\title{
Metagenomics in diagnosis and improved targeted treatment of UTI
}

Matthew Dixon (1), Maria Stefil (1), Michael McDonald (2,3), Truls Erik Bjerklund Johansen (4), Kurt Naber (5), Florian Wagenlehner (6), Vladimir Mouraviev $(2,3,7)$

(1) Norwich Medical School, University of East Anglia, UK

(2) Florida Hospital Celebration Health, Celebration, FL, USA

(3) University of Central Florida, Orlando, FL, USA

(4) Department of Urology, Oslo University Hospital, Norway

(5) Department of Urology, Technical University of Munich, Germany

(6) Department of Urology, Justus-Liebig University Giessen, Germany

(7) Central Florida Cancer Institute, Davenport, FL, USA

Corresponding author: Vladimir Mouraviev

email: mouraviev@.cfcancerinst.com

tel: +1 (919) 3708287 


\section{Abstract}

The genomic revolution has transformed our understanding of urinary tract infection (UTI). There has been a paradigm shift from the dogmatic statement that urine is sterile in healthy people, as we are becoming forever more familiar with the knowledge that bacterial communities exist within the urinary tracts of healthy people.

Metagenomics can investigate broad populations of microbial communities, analyzing all the DNA present within a sample, providing comprehensive data regarding the state of the microenvironment of a patient's urinary tract. This permits medical practitioners to more accurately target organisms that may be responsible for disease - a form of 'precision medicine'.

This paper explores the limitations of traditional methods of culture and sensitivity and delves into the recent studies involving new high-throughput genomic technologies in urological research, demonstrating the advances made in the role of urinary microbiome in a whole spectrum of pathologies from urinary tract infection, to prostate cancer. Finally, we discuss the challenges that must be overcome for such technology to become widely used in clinical practice.

\section{Keywords}

Metagenomics

Next Generation Sequencing

Urinary tract infection

Precision medicine

Targeted treatment

Infectious urology 


\section{Introduction}

The genomic revolution has transformed our understanding of urinary tract infection (UTI). There has been a paradigm shift from the dogmatic statement that urine is sterile in healthy people, as we are becoming forever more familiar with the knowledge that bacterial communities exist within the urinary tracts of healthy people. The term 'microbiota' refers to the microorganisms that exist within a niche such as the kidney, bladder, prostate etc. [1]. 'Microbiome', instead, refers to the collective microbial community occupying a reasonable well-defined habitat with its distinct physicchemical properties within their theater of activity e.g. in urine, as a result of interaction of different microbiotas. The role of these complex microbial communities is now known to be of significant importance in the pathophysiology of infection and is currently a hot topic of basic science and clinical research [1]. However, their function has not been quite so extensively studied within the realm of urological medicine.

Metagenomics allows for large quantities of DNA strands to be analyzed simultaneously, providing a wealth of information about the presence of large numbers of species in a timely fashion. One of the most attractive features of this technology to clinicians is its ability to provide much more comprehensive data regarding the state of the microenvironment of a patient's urinary tract than is yielded by a traditional culture and sensitivity (C\&S), permitting medical practitioners to more accurately target organisms that may be responsible for disease - a form of 'precision medicine'.

This paper seeks to evaluate the recent studies conducted within the urinary microbiome and different microbiotas within the urogenital system that have demonstrated the utility of the new high-throughput genomics in clinical practice, and how advances in the understanding of the role of microbiome have been made as a result of this technology.

\section{Current high-throughput genomics technologies}

Over the last two decades, the rapid development of high-throughput molecular methods to detect and characterize the enormous amount of existing bacterial, fungal, and viral pathogens within the human body enabled identification of a variety of different taxa per sample in order to diagnose the most dangerous and life-threatening "superbugs". Regarding their technical aspects, highthroughput identification methods can be classified as PCR-based quantification methods, hybridization-based methods (e.g. microarrays), second-generation fingerprinting methods e.g. RAD- seq) and sequence-based methods, e.g. metabarcoding, (meta)transcriptomics and (meta)genomics.

Also known as 'shotgun sequencing', metagenomic sequencing (MGS) can investigate the broad populations of microbial communities from complex environmental or clinical samples that helps in the discovery of novel organisms and can exemplify the dynamics of a population under different conditions [2]. All DNA present in a sample is sequenced before bioinformatic analyses are used to 
filter out the DNA that is human or from a known non-pathogenic organism in order to identify the causative agent. Usually, MGS is used to sequence the whole genome; it is a sensitive method but can produce redundant findings due to the detection of normal inhabitants of the human microbiome, rendering it difficult to interpret [3]. Next Generation Sequencing (NGS) as a branch of MGS is a more elaborate term that collectively refers to high-throughput DNA sequencing strategies that can produce robust and large amounts of genomic data in a single reaction. Additional methodologies include gene expression profiling by RNA sequencing (transcriptomics), mapping of DNA binding proteins and chromatin analysis, epigenetics, discovery of noncoding RNAs, exome sequencing, single nucleotide polymorphism detection, and amplicon resequencing [3]. NGS, also called 'massively parallel sequencing', works at a lower cost per base than the 'first generation Sanger sequencing'; these methods encompass 'second- 'and 'third-generation sequencing'. Therefore, NGS is a more commonly used term in literature, with its clinical utility discussed further in this paper. The most commonly used NGS platforms are illustrated in Figure 1 [4].

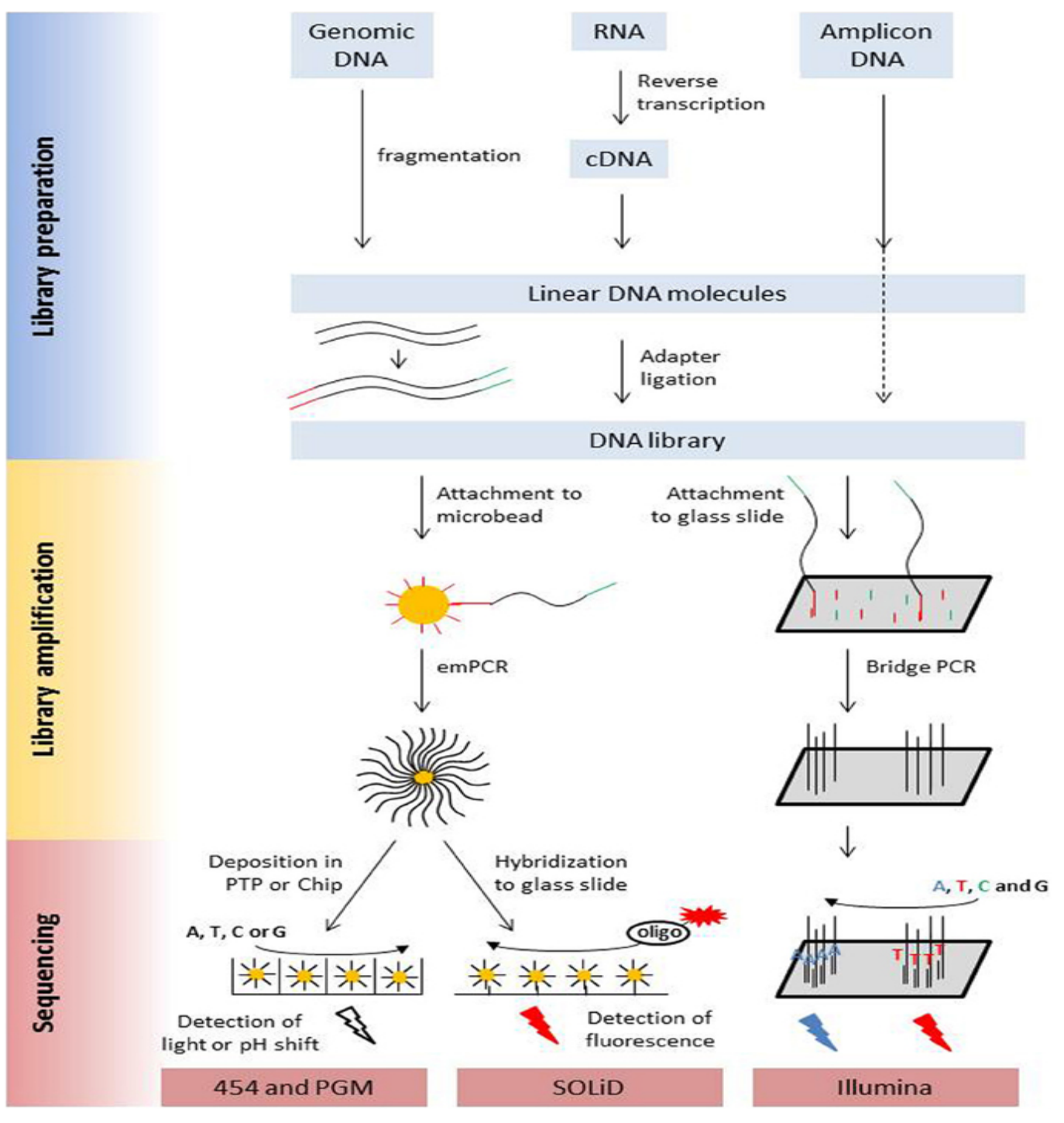


Figure 1. Scheme of step-wise approach of most commonly used NGS platforms. The primary molecules from sample are converted into double-stranded DNA which is then ligated by adapters. Adapters allow the binding of the library molecules to surfaces for subsequent amplification with separation prior to sequencing. An advanced Illumina sequencing platform uses four differently labelled nucleotides during sequencing process in multiple cycles with the desired read of length. Reprinted from "Analysis of plant microbe interactions in the era of next generation sequencing technologies," by Claudia Knief, 2014, Frontiers in Plant Science, 5, p. 216. Copyright 2014 Knief.

The 16S rRNA gene is highly conserved and found in all bacterial genomes. The specific sequences of this region are unique to each individual bacterial species. Over the last few years, a combination of PCR amplification and sequence analysis of this gene became the accepted reference standard for detection of unknown bacterial species for a single isolate, e.g. in urine sample $[5,6]$. This standard marker is a semi-quantitative assay which reports the total bacterial load as low, medium or high that corresponds to ranges for standard C\&S colony forming units (CFU) in agar plate as $<10^{5}, 10^{6}-10^{7}$, and $>10^{7}$ per $\mathrm{ml}$, respectively.

NGS can identify multiple species from a polymicrobial infection found in numerous patient specimens. NGS does not require growth, so it can identify gram-negative, gram-positive, and anaerobic bacteria as well as fungi/yeast/molds all in the same specimen using one extraction method. NGS can not only detect the bacterial species, but also delivers information about genotypic antibiotic resistance, which may be used to predict phenotypic resistance. Clinical guidance regarding antimicrobial resistance may then be provided. Genetic profiling is intended to facilitate timely management; a contrast to the 2-5-day turnaround time of C\&S [3]. Sequencing directly any clinical samples would allow NGS analysis to be conducted within one working day, even within a matter of hours depending on the specific platform that is utilized. For example, using Illumina platform, the data analysis time varies based on the number of sequence reads per sample, but the average time taken is 5 minutes per sample. Performing data analysis on the entire run takes approximately 6 hours.

While the amplification step is relatively fast, the analytic process is still time consuming. The set of data recovered is going through a bioinformatics pipeline prior to analysis. The bioinformatics pipeline takes the raw sequence data and performs cleaning, i.e. denoising. During denoising, the sequences are checked for chimeras and singletons; these sequences are removed. In addition, denoising will remove any sequences that have errors in the barcode, that don't meet at least half of the intended fragment length, and that are below our quality threshold for error rates. These sequences are deemed too low quality to provide accurate information in the target gene region. All of the high-quality sequences are then analyzed through the curated database to match against all microbes, including pathogens, normal flora, and opportunistic pathogens.

In general, NGS is currently still in its infantile stage, where phenotypic susceptibility data is extracted from a reference library of more than 25,000 specimens. It is possible, however, for clinical phenotype to be at least partially independent of genetic determinants, such as in the case 
of 'intrinsically resistant microorganisms', or frequently mutating genes [3]. Therefore, at this stage, the genotypic characteristics do not always elucidate phenotypic features with ideal concordance for the choice of treatment, especially in 'superbugs' such as multi-drug resistant Escherichia coli or Pseudomonas aeruginosa.

Other available technology that could possibly compete with NGS in terms of fast microbial detection and identification is single-and-multiplex polymerase chain reaction (PCR) combined with extended or enhanced quantitative urine culture techniques. Although multiplex PCR assays have the advantage of a rapid turnaround time (between one hour to one day) compared to NGS, specific primers are needed to amplify sequences and so their design must perpetually be updated so as to detect new variants [7]. Therefore, even a small error in the choice of primers can largely hinder antibiotic stewardship.

The following clinical case demonstrates the comparative value of standard C\&S versus PCRbased and extended C\&S (MicroGen DX PCR and MD Labs) versus NGS technologies (Volente, MicroGen ${ }^{\mathrm{DX}} \mathrm{DNA}$ ) (Table 1). This case reflects the complexity of standardization and interpretation of the results of different techniques that can dramatically affect antibiotic stewardship in the treatment of UTI. This clinical case demonstrates the superiority of NGS-based techniques over standard C\&S in that the NGS-based techniques provided a broader pathogen profile and respective pathogen load. This case also illustrates the challenges that come from heterogeneity of antibiotic resistance gene reporting by different NGS platforms. This serves to prove the substantial clinical utility and diagnostic potential that the NGS-based techniques harbor, but also highlights the necessity for standardization of the analysis and reporting of antibiograms and antibiotic resistance data. It is possible that NGS will change the way chronic infections are managed as the standard approaches were not comprehensive enough to isolate and identify a lingering infection. This case demonstrates how NGS provided a diagnostic breakthrough for the patient in question, who was suffering with recurrent UTI, something that standard diagnostic approaches had failed to achieve in the past. 


\section{Table 1. Clinical case synopsis with comparison of laboratory results.}

\section{Clinical history}

An 82-year-old lady with a history of recurrent UTI (more than three episodes of UTI per year) presented to the clinic with an acute episode after first intra-detrusor injection of Botox for her urge incontinence. She complained of dysuria, daytime frequency (10-12 times per day), nocturia (4-5 times per night), suprapubic pain, chills, rigors, and was marginally febrile. A mid-stream urine catch sample was obtained and sent for standard urinalysis, C\&S, MD laboratory for singleplex PCR for pathogen detection with extended C\&S, Volente Laboratories using multiplex PCR for both bacterial and fungal detection and susceptibility, and MicroGenDx using PCR for susceptibility and NGS for quantitative pathogen detection. Urinalysis revealed a large presence of leukocyte esterase, positive nitrites, and a small amount of red blood cells. Empiric treatment was initiated with Trimethoprim/Sulfamethoxazole 400/160mg twice per day. The results from all techniques were back within 5 days. There was a high concordance between C\&S and PCR-based techniques to detect a high load of $E$. coli $\left(>10^{5}\right.$ per $\mathrm{ml}$ ) while MD labs and Volente were able to identify an additional mixed skin flora and coagulase negative Staphylococcus species. However, NGS detected a dominant pathogen as Bacteroides fragilis (50\%) while E. coli was the second dominant species (48\%). In terms of susceptibility, the standard C\&S, MD labs and NGS revealed multi-drug resistance, including to fluoroquinolone and beta-lactam antibiotics. Primers used by Volente did not detect a quinolone resistant gene, and therefore the company recommended the use of fluoroquinolones as a treatment option. Based on the presence of quinolone resistant genes detected by NGS, a targeted antimicrobial therapy was administered using Clindamycin $300 \mathrm{mg}$ twice per day and Vancomycin $125 \mathrm{mg}$ q $6 \mathrm{hrs}$ for a total of 14 days, and alternative medications (probiotics, cranberry extract and Dmannose). By the fifth day, the patient's symptoms subsided, and upon follow-up, on day 14, she denied all symptoms. The control urine DNA sequence results demonstrated a low load of $E$. coli while all other techniques demonstrated an undetectable level of $E$. coli and other baseline pathogens.

\begin{tabular}{|c|c|c|c|}
\hline \multicolumn{4}{|c|}{ Laboratory tests } \\
\hline Test & $\begin{array}{l}\text { Microbes } \\
\text { detected }\end{array}$ & Antibiotic resistance detected & Antibiotic resistance not detected \\
\hline $\begin{array}{l}\text { Culture and } \\
\text { sensitivity }\end{array}$ & $\begin{array}{l}\text { Escherichia coli } \\
\text { (ESBL) }\end{array}$ & $\begin{array}{l}\text { Ampicillin } \\
\text { Ampicillin/Sulbactam } \\
\text { Cefazolin } \\
\text { Cefepime } \\
\text { Ceftazidime } \\
\text { Ceftriaxone } \\
\text { Cefuroxime } \\
\text { Ciprofloxacin } \\
\text { Levofloxacin } \\
\text { Tetracycline } \\
\text { Trimethoprim/Sulfamethoxazole }\end{array}$ & $\begin{array}{l}\text { Amikacin } \\
\text { Amoxicillin/ Clavulanate } \\
\text { Ertapenem } \\
\text { Gentamicin } \\
\text { Imipenem } \\
\text { Meropenem } \\
\text { Nitrofurantoin } \\
\text { Piperacillin/Tazocin } \\
\text { Tobramycin }\end{array}$ \\
\hline MD Labs & $\begin{array}{l}\text { Escherichia coli } \\
\text { (ESBL) }\end{array}$ & $\begin{array}{l}\text { Amoxicillin/Clavulanate } \\
\text { Ampicillin } \\
\text { Cefazolin } \\
\text { Cefepime } \\
\text { Ceftriaxone } \\
\text { Ciprofloxacin } \\
\text { Levofloxacin } \\
\text { Tetracycline } \\
\text { Trimethoprim/Sulfamethoxazole }\end{array}$ & $\begin{array}{l}\text { Ertapenem } \\
\text { Gentamycin } \\
\text { Nitrofurantoin } \\
\text { Piperacillin/Tazobactam }\end{array}$ \\
\hline \multirow[t]{2}{*}{ Volente } & Escherichia coli & \multirow{2}{*}{$\begin{array}{l}\text { Class A beta-lactamases (CTX- } \\
\text { M1, M2, M9, M8/25 groups) } \\
\text { Macrolide } \\
\text { Lincosamide } \\
\text { Streptogramin } \\
\text { Tetracycline } \\
\text { Trimethoprim/Sulfamethoxazole }\end{array}$} & $\begin{array}{l}\text { AmpC beta-lactamases } \\
\text { Class A beta-lactamases (SHV, KPC groups) } \\
\text { Class B metallo-beta-lactamases }\end{array}$ \\
\hline & $\begin{array}{l}\text { Staphylococcus } \\
\text { (coagulase } \\
\text { negative: } \\
\text { epidermidis, }\end{array}$ & & $\begin{array}{l}\text { Class D oxacillinases } \\
\text { Fluoroquinolone } \\
\text { Methicillin } \\
\text { Minor extended spectrum beta-lactamases } \\
\text { Vancomycin }\end{array}$ \\
\hline
\end{tabular}




\begin{tabular}{|c|c|c|c|c|}
\hline & $\begin{array}{l}\text { haemolyticus, } \\
\text { lugdunensis, } \\
\text { saprophyticus) }\end{array}$ & & & \\
\hline \multirow{3}{*}{$\begin{array}{l}\text { MicroGen }^{\mathrm{DX}} \\
\text { PCR }\end{array}$} & Escherichia coli & \multirow{3}{*}{$\begin{array}{l}\text { Aminoglycoside } \\
\text { Beta-lactam (except } \\
\text { carbapenem) } \\
\text { Methicillin } \\
\text { Quinolone }\end{array}$} & \multirow{3}{*}{\multicolumn{2}{|c|}{$\begin{array}{l}\text { Carbapenem } \\
\text { Macrolide } \\
\text { Tetracycline } \\
\text { Vancomycin }\end{array}$}} \\
\hline & Prevotella bivia & & & \\
\hline & $\begin{array}{l}\text { Enterococcus } \\
\text { faecalis }\end{array}$ & & & \\
\hline \multirow{2}{*}{$\begin{array}{l}\text { MicroGen DX } \\
\text { DNA } \\
\text { sequence }\end{array}$} & $\begin{array}{l}\text { Bacteroides } \\
\text { fragilis }(50 \%)\end{array}$ & & \multicolumn{2}{|c|}{$\begin{array}{l}\text { Recommended antibiotics from library: } \\
\text { - B. fragilis: }\end{array}$} \\
\hline & $\begin{array}{l}\text { Escherichia coli } \\
(48 \%)\end{array}$ & & $\begin{array}{r}\circ \\
\circ \\
\circ \\
-\quad \text { E. col } \\
\circ \\
\circ \\
\circ \\
\circ\end{array}$ & $\begin{array}{l}\text { Doripenem } \\
\text { Clindamycin } \\
\text { Tetracycline } \\
\text { Trimethoprim/Sulfamethoxazole } \\
\text { Nitrofurantoin } \\
\text { Vancomycin } \\
\text { Linezolid }\end{array}$ \\
\hline
\end{tabular}

*Bolded text reflects differences of susceptibility or resistance to certain antibiotics between different techniques.

\section{Clinical application of NGS in urological medicine}

A limited number of trials have been found in the current body of literature regarding urinary tract microbiome.

\section{Urinary Tract Infection}

In a phase II study by McDonald et al. examining the diagnosis and treatment of acute uncomplicated and complicated cystitis, symptom scores were significantly better for those patients whose treatment was based on NGS results as opposed to traditional C\&S [8]. All 44 patients showed positive results in DNA sequencing tests, while only 13/44 patients had positive urine culture tests. In patients who showed negative culture results, but positive NGS results, symptoms improved when they started treatment based on the results of NGS. Although this study was of a small size, it shows that NGS may be of great use, particularly in patients who continue to experience symptoms after typical empirical antibiotic therapy. Furthermore, anaerobic bacteria were detected in 20 of 44 patients via NGS - culpable for UTI symptoms in 10 of these patients. This appears to be yet another benefit of NGS, as anaerobic bacteria have classically been missed in cases of UTI due to the difficulty in detecting them via culture methods.

Wagenlehner et al demonstrated that $141 / 588$ patients (24\%) with typical symptoms of acute lower uncomplicated UTI and pyuria had negative urine cultures i.e. $<103 \mathrm{CFU} / \mathrm{mL}$, furthering our 
understanding of the limitations of urine cultures in their ability to detect clinically important infections [9].

\section{Prophylaxis of UTI following transrectal biopsy}

Elshal et al compared the post-transrectal biopsy infectious complication (TBIC) rate between patients separated into standard ( 3 days $500 \mathrm{mg}$ ciprofloxacin twice daily, beginning the night before the biopsy), augmented (ciprofloxacin and single 160mg gentamicin IM), and targeted prophylaxis (based on results of rectal swab cultures). There was a lower rate of TBICs among the patients allocated to the augmented prophylaxis group, regardless of the variation in the number of biopsy cores taken when compared to the other two groups [10].

Mouraviev et al. evaluated NGS in rectal swabs of 50 patients before prostate biopsy. Multiple species were detected in these rectal swab samples with a median of 9 pathogens (range 1-16) [11]. In 35 of $50(70 \%)$ cases multiple drug resistance genes were detected, and $24(48 \%)$ of those were resistant to fluoroquinolones. Empiric prophylaxis was then modified from levofloxacin to NGS DNA-guided targeted antibiotic(s). In 17 cases, fungal species were detected, 11 of whom harbored multiple fungal spp. That was an indication to supplement prophylaxis with an antifungal agent. This strategy was associated with avoidance of serious infectious complications in all patients within 30 days after biopsy.

\section{Prostate Cancer}

Currently, it is thought that chronic inflammation secondary to prostate infection may play a role in the development and progression of prostate cancer [12].

Using NGS, Shrestha et al analyzed 135 urine samples which had been obtained post digital rectal examination [13]. Overall, there was no significant disparity in the variety of microbial populations between men with prostate cancer and men without, and no evidence of a connection between bacterial load, PSA levels, or the degree of inflammation seen on biopsy. Despite this, a group of bacteria were observed in this study which disproportionately existed in patients with cancer. Notably, these included Anaerococcus spp., among others. The importance of the detection of these species is that all of them have been suggested as organisms responsible for conditions such as prostatitis and UTI.

Frugé et al looked at the dietary changes which impact the gut microbiota in overweight and obese men with prostate cancer [14]. Analysis of the microbiome from fecal samples revealed some 'unexpected correlations with several Firmicutes and Proteobacteria genera and biopsy Gleason sum at diagnosis' were detected. Their work indicates that a possible 'microbiome signature of aggressive prostate cancer' exists, although further research must be done to determine the appearance of this signature, and whether it can be altered to govern the development and spread of prostate cancer in the future. 
Cavarretta et al found that genera such as Staphylococcus spp. were more prevalent in samples of tumor tissue and, conversely, bacteria within the order of Lactobacillales, notably Streptococcus spp. were significantly less common in samples of tumor tissue [15]. This supports the notion that Streptococcus spp. may exist in the normal microbiome of the prostate and may be of benefit to health. Here, NGS was used to detect species which previously may not have been detected due to the lack of sensitivity of traditional cultures.

\section{Chronic Pelvic Pain Syndrome (CPPS)}

CPPS constitutes a large majority of 'prostatitis' cases and is typified by symptoms which last for at least 3 months within a 6-month period [16]. Urinary symptoms and sexual dysfunction in the absence of urinary tract bacterial infection is classical. So far, the etiology is not well understood and is thought to be heterogeneous. Shoskes et al analyzed DNA from fecal samples and found that there was a significantly less diverse population of microbes present within the guts of patients diagnosed with CPPS. Furthermore, there was evidence to suggest that there are lower amounts of several taxa of bacteria among patients with CPPS. Of particular interest is that quantities of organisms within the genera Prevotella are sufficiently low among patients with CPPS to potentially act as a biomarker and be a target for manipulation in the future as a means of treating this common condition [17].

\section{Urgency Urinary Incontinence}

Karstens et al revealed that there is a disparity in the prevalence of numerous groups of bacteria between patients with urgency urinary incontinence and those without. The results of this study also showed that there is a positive correlation between reduced microbial diversity and worsening symptoms associated with urgency urinary incontinence [18]. Within this context, it is also thought that microbiota has a role in a patient's response to treatments such as anticholinergics i.e. female patients in whom there are smaller numbers of Lactobacillus may be more likely to suffer from a post-treatment UTI [19]. Several studies have so far demonstrated a similar picture in that a patient's response to treatment may be affected by the diversity of bacteria and the abundance of particular groups $[20,21]$.

\section{Neurogenic Bladder}

Using NGS, Fouts et al found that there are differences in the microbiome between patients with neurogenic bladder dysfunction and those with healthy bladder function. The results showed that there are higher levels of Lactobacillus and Corynebacterium in healthy bladders, and in patients with neurogenic bladder disorder, there is a tendency for higher levels of Escherichia, Enterococcus, and Klebsiella [22].

Mouraviev et al presented at the last American Urology Association 2018 meeting the findings of NGS of urine samples from their cohort of 13 patients who all had positive bacterial or fungal 
pathogens with catheter-associated UTI [23]. The median number of organisms present in each specimen was 3 species. The majority of patients had a high bacterial load $\left(>10^{7}\right.$ microorganisms per $\mathrm{ml}$ ). Resistance genes to different antibiotics detected were found in 9/13 samples and 6 of them had multidrug resistance genes. Of note, a standard culture in 3 patients failed to detect a dominant pathogen that was successfully detected by NGS and required a different targeted treatment. Fungi were detected in the samples of 2 patients.

\section{Limitations of NGS}

In order for NGS to become a routine form of diagnosis for infectious urological diseases, formal training in bioinformatics for clinical microbiologists would need to be considered, as would the development of secure, comprehensive databases, and the methodology of clinical reporting would have to be thought through clearly $[24,25,26]$.

NGS remains a highly sensitive technique which is able to identify a whole bacterial and fungal association including normal inhabitants (commensal) of urine microbiome maintaining a reasonable balance and protection against aggressive pathogens [27]. Evidently, as more bacterial strains are detected, a clinical problem of determining which pathogen is responsible for UTI in every individual case will arise. It is also of crucial importance to distinguish pathogens from other contaminating or commensal flora that can be mistakenly treated with antibacterial therapy. How we can distinguish a clinically relevant pathogen from bystanders remains a challenging task of the whole analysis. There is a lot of work on standardization and creation of prediction algorithms need to be performed. So far, all microbes are reported to the physician because they provide useful information on the microbial community in the infection. The normal flora provides information about how the pathogens compare to the normal flora. For example, the community of bacteria in the vagina should have Lactobacillus. If Lactobacillus is present, the physician can use this information to compare the percentage of Lactobacillus (healthy flora) with the percentage of Atopobium parvulum to view the shift in the community and treat the pathogen. In addition, the information can be used to see the opportunistic pathogens that only play a role in disease in the presence of other organisms. For instance, Staph epidermidis is a commensal flora of skin, but once a wound breaks the barrier of the skin, then it can cause infection and should be something considered by the physician when deciding on the treatment regimen.

Therefore, the quantitative and qualitative metrics to evaluate a baseline bacterial and fungal profile in normal individuals and UTI patients have to be introduced and validated in multiinstitutional trials to collect more robust data [28]. Assay validation will be a critical step in assessing performance, and it is imperative that standard operating procedures are constructed in order to produce accurate results [29]. Laboratories will need to provide independent verifications of test performance set side by side with other laboratories in order to ensure proficiency. This quality control reveals another impediment to the introduction of NGS technology - the need for a reference material and data set against which laboratories can assess their performance. At this 
point in time, there are no such formal materials available, though they are currently in development.

With regards to the reference library of genomic data, we realize that most of the data already present in the databases available may not be of good quality. Therefore, it is important to note that sequences and genomes can, and indeed have been poorly annotated, with some annotations being incorrect. The public available database like NCBI do suffer from poor and incorrect annotation, that can significantly compromise all the future developments. Since almost anyone can upload sequences to the database, they often only classify the sequence reads down to the taxonomy that the researcher decides, so there will be a lot of reads classified as thermal vent bacteria instead of the genus and species. In addition, some researchers will upload sequences that initially didn't match any sequence in their subset of research, but they fail to check the sequences against the entire database, so they falsely call the sequence novel and provide it with a new name. Those are just a few of the problems that public available databases face. This is not inconsequential, as identification of responsible pathogens heavily relies on the accuracy of such a database and, as such, it is crucial that only sequences with a known origin are incorporated into these databases. For example, in our opinion, the database at MicroGen DX, one of the first NGS companies in the U.S.A, is heavily curated before being used for patient testing. For sequences to be entered into this database, they must go through several stages of quality verification. First, the sequences must cover genes of interest. Then, the sequences must be annotated in the proper format. If the annotation is incomplete, then they will not be incorporated for verification. Next, the sequences will be tested for accuracy by verifying that the sequences are attributed to the correct species. Any new sequence such as the ones called novel but match a current strain (i.e. incorrectly annotated), will be found during the accuracy check and incorporated under the proper name. The sequences are then run against a test set of species that have been sequenced in house from ATCC standards to ensure that the proper identification of these known positives has not been skewed. Lastly, any taxonomy changes to previous or current species are updated in the database. This curation occurs every 6 months. Such strict rules and standards should be approved and implemented by official regulatory organizations and institutions responsible for future implementation of metagenomics.

As with most novel technologies, start-up costs of NGS platforms are likely to be substantial, but maintenance costs and the labor expenses of a well-trained technologist would also need to be accounted for. Despite these charges, NGS still runs at a significantly lower cost per base than Sanger sequencing [30]. With that said, NGS is relatively expensive when compared to technology such as matrix-assisted laser desorption ionization time of flight mass spectrometry (MALDI-TOF MS) [3]. MALDI-TOF is an interesting competitor for rapid microbial identification, but it also suffers drawbacks. To use MALDI-TOF, the bacteria and fungi must be cultured in order to obtain intact organisms and remove the human host cells. It also requires one microbe to be analyzed at once in order to see the specific separation related to one microbe rather than a mixture of microbes. Since the species have to be cultured/grown first, any species that cannot be grown or has a lot of difficulty growing will either be missed or delay the identification. In addition, the cells must be intact, so the specimen's age and storage conditions will limit its utility. MALDI-TOF compares the 
microbial characteristics to a database and anything that is not added or poorly annotated to the database will not be identified or will be identified incorrectly. This is particularly problematic for anaerobes and fungi which are poorly characterized in most MALDI-TOF commercially available databases. MALDI-TOF also have trouble with low bacterial load specimens due to the low level of intact organisms present. They combat this by using culture as a way to multiply the organisms, but culture takes time and not all species can be easily grown. As we mentioned above, NGS takes 3-5 days to complete the entire process from extraction to data analysis; however, if you include the growth time to the data analysis time for MALDI-TOF, the sample to answer time is not very different especially in difficult-to-identify species.

The question of how to manage and store the copious amount of data produced is a crucial one; the sheer quantity of raw data involved renders its long-term storage troublesome [3]. Data can be stored in files that are specific to the NGS platform, but this is complicated due to the heterogeneity of the formats available. One way of circumventing this problem would be to use cloud-based storage systems, standardizing the format of the data produced by differing NGS platforms. However, laboratories would have to be extremely diligent, ensuring that the appropriate controls are in place to maintain patient confidentiality.

\section{Conclusion}

Until recently, the human urinary tract was believed to be a sterile region of the body when in good health. With the use of NGS, there is now a great deal of evidence to the contrary. The urinary tract possesses a microbiome and, with this new technology now available, it is possible to explore its role in pathology and, conversely, the impact of disease on the microbiome itself.

Prior to the development of NGS, the main method of studying microbial communities involved attempting to culture the organisms that may exist within a sample. With the knowledge that the microbiome is an important piece of the urology puzzle, we have to be mindful of the limitations of cultures, and how they regularly have the effect of overlooking infections which may very well be crucial.

Although NGS is at an early stage of its development, its ability to quickly detect and identify the entire spectrum of microbes present within a sample with accuracy, and its capacity to predict phenotypic resistance patterns via genomic data proves its superiority to the slower, traditional methods of culture and sensitivity. However, at this point in time, there are still limitations in precisely defining leading pathogen(s) which can contribute to the development of UTI and sufficiently distinguish them from other contaminating or commensal strains. The implementation of NGS in clinical laboratories will certainly demand a great deal of careful thought to ensure patient confidentiality while simultaneously storing data in a manner which will optimize public benefit.

The further evolution of NGS will be one towards truly individualized patient care, allowing preemptive diagnoses in the asymptomatic population, and predictions to be made about a patient's 
likelihood of developing severe UTI and design an actionable and targeted antibiotic stewardship based on a patient's microbiome and their pharmacogenetics.

With a lower cost per base than competing technologies, combined with its potential for revealing the intricacies of the relationship between microbiota and the human body, NGS appears to be the future of diagnosis, prevention, and precision treatment of a number of urological conditions.

\section{Acknowledgements}

The authors would like to thank Jennifer White, M.S., MB(ASCP)cm for her excellent technical assistance and thorough description of metagenomics and Next Generation Sequence technologies.

\section{Authors' contributions}

M Dixon: Manuscript writing/editing

M Stefil: Manuscript writing/editing

M McDonald: Principal Investigator of clinical trial; interpretation of NGS results; manuscript writing/editing

TE Bjerklund Johansen: Diagnostic results analysis; manuscript writing/editing

K Naber: Diagnostic results analysis; manuscript writing/editing

F Wagenlehner: Diagnostic results analysis; manuscript writing/editing

V Mouraviev: Project development; interpretation of NGS results; manuscript writing/editing

\section{Compliance with Ethical Standards}

\section{Funding}

None.

\section{Conflicts of interest}

Dr Mouraviev is a consultant for MicroGenDx. 


\section{Informed consent:}

Informed consent was obtained from all individual participants included in the study.

\section{References}

1. Mouraviev V, McDonald MW (2018) An implementation of next generation sequencing for prevention and diagnosis of urinary tract infection in urology. Can J Urol 25:9349-9356

2. Forbes JD, Knox NC, Ronholm J et al (2017) Metagenomics: The Next Culture-Independent Game Changer. Front Microbiol. 8:1069

3. American Society for Microbiology (2016) Applications of Clinical Microbial Next-Generation Sequencing: Report on an American Academy of Microbiology Colloquium held in Washington, DC, in April 2015. American Academy of Microbiology https://www.ncbi.nlm.nih.gov/books/NBK513764/ Accessed 1 December 2018.

4. Knief C (2014) Analysis of plant microbe interactions in the era of next generation sequencing technologies. Front Plant Sci 5:206

5. Rizzo JM, Buck MJ (2012) Key principles and clinical applications of "Next-Generation" DNA sequencing. Cancer Prevention Research 5:887-900

6. Wellinghausen N, Kochem AJ, Disqué C et al (2009) Diagnosis of bacteremia in wholeblood samples by use of a commercial universal 16S rRNA gene-based PCR and sequence analysis. J Clin Microbiol 47:2759-2765

7. Prachayangprecha S, Schapendonk CM, Koopmans MP et al (2014) Exploring the potential of next-generation sequencing in detection of respiratory viruses. J Clin Microbiol 52:37223730

8. McDonald MW, Kameh D, Johnson ME et al (2017) A Head-to-Head Comparative Phase II Study of Standard Urine Culture and Sensitivity Versus DNA Next-generation Sequencing Testing for Urinary Tract Infections. Rev Urol 19:213-220

9. Wagenlehner FM, Abramov-Sommariva D, Höller M et al (2018) Non-Antibiotic Herbal Therapy (BNO 1045) versus Antibiotic Therapy (Fosfomycin Trometamol) for the Treatment of Acute Lower Uncomplicated Urinary Tract Infections in Women: A Double-Blind, ParallelGroup, Randomized, Multicentre, Non-Inferiority Phase III Trial. Urol Int 101:327-336

10. Elshal AM, Atwa AM, El-Nahas AR et al (2018) Chemoprophylaxis during transrectal prostate needle biopsy: critical analysis through randomized clinical trial. World $\mathrm{J}$ Urol 36:1845-1852

11. Mouraviev V, McDonald MW, Skinner C et al (2018) MP15-14 the value of next generation dna sequencing testing in rectal swabs before transrectal prostate biopsy for individual and targeted prophylaxis of urinary tract infection. J Urol 199(4):e194

12. Sfanos KS, Isaacs WB, and De Marzo AM (2013) Infections and inflammation in prostate cancer. Am J Clin Exp Urol. 1:3-11 
13. Shrestha E, White JR, Yu SH et al (2017) Profiling the Urinary Microbiome in Men with Positive versus Negative Biopsies for Prostate Cancer. J Urol 199:161-171

14. Frugé AD, Ptacek T, Tsuruta Y et al (2018) Dietary Changes Impact the Gut Microbe Composition in Overweight and Obese Men with Prostate Cancer Undergoing Radical Prostatectomy. J Acad Nutr Diet 118:714-723.e1

15. Cavarretta I, Ferrarese R, Cazzaniga W et al (2017) The Microbiome of the Prostate Tumor Microenvironment. Eur Urol 72:625-631

16. Krieger JN, Nyberg L Jr, Nickel JC (1999) NIH consensus definition and classification of prostatitis. JAMA 282:236-7

17. Shoskes DA, Wang H, Polackwich AS et al (2016) Analysis of Gut Microbiome Reveals Significant Differences between Men with Chronic Prostatitis/Chronic Pelvic Pain Syndrome and Controls. J Urol 196:435-441

18. Karstens L, Asquith M, Davin S et al (2016) Does the urinary microbiome play a role in urgency urinary incontinence and its severity? Front Cell Infect Microbiol 6:78

19. Pearce MM, Zilliox MJ, Rosenfeld AB et al (2015) The female urinary microbiome in urgency urinary incontinence. Am J Obstet Gynecol 213:347.e1-11

20. Brubaker L, Nager CW, Richter HE et al (2014) Urinary bacteria in adult women with urgency urinary incontinence. Int Urogynaecol J 25:1179-84

21. Pearce MM, Hilt EE, Rosenfeld $A B$ et al (2014) The female urinary microbiome: a comparison of women with and without urgency urinary incontinence. MBio. 5:e01283-14

22. Fouts DE, Pieper R, Szpakowski S et al (2012) Integrated next-generation sequencing of $16 S$ rDNA and metaproteomics differentiate the healthy urine microbiome from asymptomatic bacteriuria in neuropathic bladder associated with spinal cord injury. J Transl Med 10:174

23. Mouraviev V, McDonald MW (2018) MP23-06 An utilization of next generation sequencing of urine samples for monitoring of urinary tract infection in patients with neurogenic bladder. J Urol 199:e283

24. Smelov V, Naber K, Bjerklund Johansen TE (2016) Improved Classification of urinary tract infection: Future consideration. Eur Urol Suppl 15:71-80

25. Hiergeist A, Gessner A (2017) Clinical implications of microbiome in urinary tract diseases. Curr Opin Urol 27:93-98

26. Pak TR, Kasarkis A (2015) How Next-Generation Sequencing and multiscale data analysis will transform infection disease management. Clin Infect Dis 61:1695-1702

27. Smelov V, Naber K, Bjerklund Johansen TE (2016) Letter to the editor: Diagnostic criteria in urological diseases do not always match with findings by extended culture techniques and metagenomics sequencing of 16S rDNA. Open Microbiol J 10:23-26

28. Davenport M, Mach KE, Shortliffe LMD et al (2017) New and developing diagnostic technologies for urinary tract infection. Nat Rev Urol 14;296-310

29. Onsongo G, Erdmann J, Spears MD et al (2014) Implementation of cloud based next generation sequencing data analysis in a clinical laboratory. BMC Res Notes 7:314 30. Aziz N, Zhao Q, Bry L et al (2015) College of American Pathologists' laboratory standards for next-generation sequencing clinical tests. Arch Pathol Lab Med 139:481-493 